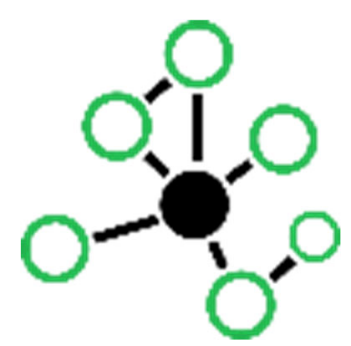

\title{
Big Data Analytics on combining RADAR and optical remote sensing imagery
}

\author{
Mirt, A. ${ }^{1}$, J. Reiche ${ }^{1}$, M. Herold ${ }^{1}$, J. Verbesselt $^{1}$ \\ ${ }^{1}$ Laboratory of Geo-information Science and Remote Sensing, Wageningen University \& Research, Droevendaalsesteeg \\ 3, 6708PB Wageningen, the Netherlands \\ Corresponding author's e-mail: andrei.mirt@wur.nI
}

Remote sensing data is big, which makes it inherently not FAIR, due to the great burden put on the users, for the data to be truly Accessible. In turn, this makes it unfeasible to process data to an Interoperable form. The ESA's Copernicus program raised the bar for quality data in earth observation satellite sensors through the Sentinel satellites. The new data is driving a revolution in Earth Observation research through its large volume, but also through its open and free design. Dense RADAR (Sentinel-1), and optical (Sentinel-2) satellite image time series can support services and science better at a global scale. We have developed new approaches to combine optical and RADAR data for rapid change monitoring, which have been tested in applications like illegal deforestation activities in the tropics. Technical key data science challenges remain, which prevent scientists and commercial users to tackle large area monitoring and service development. Firstly, efficient and scalable methods to make optical and RADAR satellite images interoperable are not available. Secondly, a solution to truly access and process large amounts of Sentinel satellites is missing. Our research will address these key challenges and will build on our existing algorithms and expertise. Together with key Dutch end users we will apply and test our developments for two usecases focusing on Sentinel-based rapid detection of forest change in the tropics and land cover monitoring in the Netherlands. 